\title{
On the Structural Phase Transition in a Perovskite-Type Diaminopropanetetrachlorocuprate(II) $\mathrm{NH}_{3}\left(\mathrm{CH}_{2}\right)_{3} \mathrm{NH}_{3} \mathrm{CuCl}_{4}$ Crystal
}

\author{
O. Czupiński ${ }^{a}$, A. Ingram ${ }^{b}$, M. Kostrzewa $^{b}$, J. Przesławski ${ }^{c}$ And Z. Czapla ${ }^{b, c}$ \\ ${ }^{a}$ Faculty of Chemistry, University of Wrocław, F. Joliot-Curie 14, 50-383 Wrocław, Poland \\ ${ }^{b}$ Department of Physics, Opole University of Technology, Ozimska 75, 45-271 Opole, Poland \\ ${ }^{c}$ Institute of Experimental Physics, University of Wrocław, Pl. M. Borna 9, 50-204 Wrocław, Poland
}

(Received December 13, 2016; in final form February 11, 2017)

\begin{abstract}
Chemical preparation, differential scanning calorimetry and thermal stability differential thermal gravimetry studies, positron annihilation lifetime investigations, optical observations as well as electric properties of the $\mathrm{NH}_{3}\left(\mathrm{CH}_{2}\right)_{3} \mathrm{NH}_{3} \mathrm{CuCl}_{4}$ crystal are presented. On the basis of the differential scanning calorimetry response the structural phase transition of the first order was observed at $436 \mathrm{~K}$. The enthalpy and entropy of the phase transition are equal to $1120 \mathrm{~J} / \mathrm{mol}$ and $2.57 \mathrm{~J} /(\mathrm{mol} \mathrm{K})$, respectively. Differential thermal analysis and thermogravimetric analysis studies confirmed the phase transition at $436 \mathrm{~K}$ and one can conclude the chemical and thermal stability of the compound up to about $480 \mathrm{~K}$. Optical observations showed a continuous change of colour from yellow to dark brown above the phase transition to $436 \mathrm{~K}$. Dielectric measurements showed a significant increase of conductivity upon approaching the phase transition regions, with a significant increase above the phase transition temperature. An activation energy dependent on the temperature range, and different for each particular phase, is obtained from measurements of complex impedance.
\end{abstract}

DOI: 10.12693/APhysPolA.131.304

PACS/topics: 62.20.D-, 77.22.Gm, 78.70.Bj

\section{Introduction}

The properties and structural phase transition of hybrid organic-inorganic compounds are related to their structures and the interaction of cationic units with complex anionic sublattices. Among them an interesting group of hybrid compounds are perovskite-type layered compounds containing propylenediammonium cations $\left(\mathrm{NH}_{3}\left(\mathrm{CH}_{2}\right)_{3} \mathrm{NH}_{3}\right)$ and layered metal-halogen anionic sublattices. Up to now, structural phase transitions were found in the case of crystals containing propylenediammonium cations in $\left[\mathrm{NH}_{3}\left(\mathrm{CH}_{2}\right)_{3} \mathrm{NH}_{3}\right] \mathrm{CdCl}_{4}-$ phase transition at $375 \mathrm{~K}[1-3]$ and $\left[\mathrm{NH}_{3}\left(\mathrm{CH}_{2}\right)_{3} \mathrm{NH}_{3}\right] \mathrm{CdBr}_{4}$ - phase transitions at $326 \mathrm{~K}$ and $385 \mathrm{~K}$ [4, 5]. Two structural phase transitions were found at $305 \mathrm{~K}$ and $337 \mathrm{~K}$ in $\left[\mathrm{NH}_{3}\left(\mathrm{CH}_{2}\right)_{3} \mathrm{NH}_{3}\right] \mathrm{MnCl}_{4}$ [6]. The $\left[\mathrm{NH}_{3}\left(\mathrm{CH}_{2}\right)_{3} \mathrm{NH}_{3}\right] \mathrm{CuCl}_{4}$ (abbreviated as DAPCCl) crystal is orthorhombic $[7,8]$ and isomorphous with $\left[\mathrm{NH}_{3}\left(\mathrm{CH}_{2}\right)_{3} \mathrm{NH}_{3}\right] \mathrm{CdCl}_{4}$ at the room temperature phase [1]. Two successive phase transitions are proposed to appear at $334 \mathrm{~K}$ and $433 \mathrm{~K} \mathrm{[8]}$ and the transition at $433 \mathrm{~K}$ is regarded as irreversible. The aim of our study is to grow the crystal, check and characterize the observed phase transitions in more detail and to study some physical properties of the single crystal. In addition we pay attention to the reversibility of the phase transition at $433 \mathrm{~K}$. To characterize the phase transition we used several experimental methods, i.e. differential scanning calorimetry (DSC), differential thermal analysis (DTA), thermogravimetric analysis (TGA), polarized microscopic observation, dielectric techniques, and positron annihilation.

\section{Experimental}

The polycrystals of $\mathrm{NH}_{3}\left(\mathrm{CH}_{2}\right)_{3} \mathrm{NH}_{3} \mathrm{CuCl}_{4}$ (DAPCCl) were prepared from stoichiometric quantities of cuprum chloride and propylenediammonium chloride with a small excess of hydrochloric acid to avoid hydrolysis of the substance. Single crystals were prepared from aqueous solutions by slow evaporation method at a constant temperature of $295 \mathrm{~K}$. Slow evaporation allows one to obtain sufficiently good quality single crystals with a form of thin plates. The thickness of the crystal was not bigger than $1.5 \mathrm{~mm}$. At room temperature the crystals were yellowgreen (yellow in transmitted light). Thermal studies were done using a Perkin-Elmer 8500 DSC at heating-cooling rates of $20 \mathrm{~K}$ and $10 \mathrm{~K} / \mathrm{min}$ in the temperature range 250-500 K. DTA and TGA were carried out by means of a Seteram SETSYS 16/18 instrument in the temperature range $295-780 \mathrm{~K}$ on a heating run at the rate of $2 \mathrm{~K} / \mathrm{min}$. For each measurement a new piece of dry single crystal was used.

Dielectric measurements were performed by means of impedance spectroscopy using a computer controlled Hioki 3532-50 LCR impedance analyzer at a frequency of $200 \mathrm{~Hz}$ to $5 \mathrm{MHz}$ and a temperature range $293-440 \mathrm{~K}$. The rate of heating/cooling was $0.25 \mathrm{~K} / \mathrm{min}$ in the vicinity of the phase transition. The surfaces of the samples were covered with Hans Wolbring $\mathrm{GmbH}$ carbon paste electrodes instead of silver paste electrodes to avoid any chemical reaction. Measurements were done on the samples along the $b$ crystallographic axis. As the crystals were rather thin and very fragile, we were unable to prepare the samples along the $a$ - and $c$-axes suitable for die- 
lectric studies. The best optical quality crystalline plates were used in all our experimental studies.

Positron annihilation lifetime (PAL) measurements were obtained using a conventional fast-fast coincidence ORTEC system of 230 ps resolution defined as halfwidth of peak maximum for single Gaussian of a standard ${ }^{60} \mathrm{Co}$ source. The measurements were performed at a temperature $T=295 \mathrm{~K}$ and relative humidity $\mathrm{RH}=35 \%$. About $10^{6}$ elementary annihilation events were accumulated to extract positron lifetimes $\tau_{1}, \tau_{2}$ and intensities $I_{1}, I_{2}$ from raw PAL spectra fitted by two components. The mathematical treatment was performed with the LT 9.0 program [9]. The source contribution was evident at the level of $12 \%$, allowing compensation of input from positrons annihilated in the Kapton foil $\left(\tau_{s}=0.372 \mathrm{~ns}\right)$. Chemical analysis was done for as-grown (the fresh) crystal and for the crystal heated up to $443 \mathrm{~K}$ and cooled down to room temperature.

\section{Results of experimental measurements and discussion}

\subsection{DSC studies}

The dependence of the heat flow for $\mathrm{NH}_{3}\left(\mathrm{CH}_{2}\right)_{3} \mathrm{NH}_{3} \mathrm{CuCl}_{4}$ is shown in Fig. 1 .

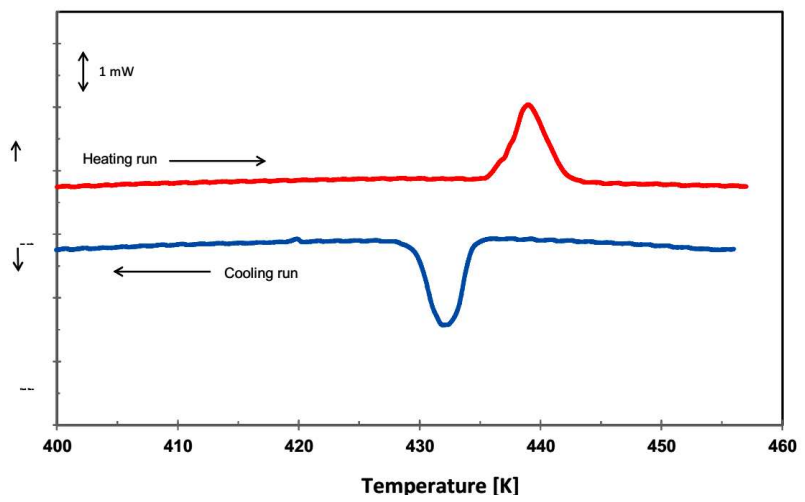

Fig. 1. The heat flow as a function of temperature for the $\mathrm{NH}_{3}\left(\mathrm{CH}_{2}\right)_{3} \mathrm{NH}_{3} \mathrm{CuCl}_{4}$ crystal for the cooling and heating runs.

As seen in Fig. 1 an anomaly of the heat flow is observed with a maximum at $439 \mathrm{~K}$ on heating and at $432 \mathrm{~K}$ on cooling. The onsets are found at $436 \mathrm{~K}$ (heating) and $434 \mathrm{~K}$ (cooling). The shape of the anomaly is characteristic for the first-order phase transition. The anomaly is reversible without any remarkable changes in repeated successive heating/cooling runs. The average enthalpy and entropy of phase transition estimated from DSC results are equal to $1120 \mathrm{~J} / \mathrm{mol}$ and $2.57 \mathrm{~J} /(\mathrm{mol} \mathrm{K})$, respectively. The estimated transition entropy indicates the displacive character of the phase transition. This value is smaller than that expected in the case of an order-disorder transition with two equilibrium positions described by the well known formula $\Delta S=R \ln 2=5.76 \mathrm{~J} /(\mathrm{mol} \mathrm{K})$. The temperature of this transition is in agreement with the temperature presented in [8] although the shape of DSC anomaly presented in [8] is very broad and strange (not characteristic for a phase transition). The enthalpy and entropy for this transition are smaller in comparison to those observed in [8]. We do not observe any traces of the phase transition at about $334 \mathrm{~K}$ in comparison to the observation in [8] where a large thermal anomaly was observed. From the DSC studies one can distinguish two phases, namely the phase I below $436 \mathrm{~K}$ and the phase II above $436 \mathrm{~K}$.

\subsection{DTA and TGA}

To make sure that the anomaly observed in the DSC is consistent with the structural phase transition, DTA and TGA were performed. The observed DTA and TGA signals are presented in Fig. 2.

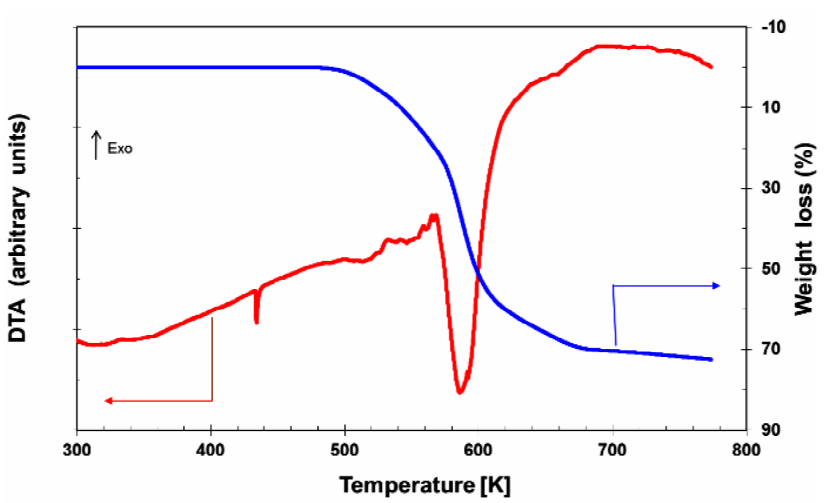

Fig. 2. The DTA and TGA signals as a function of a temperature for the $\mathrm{NH}_{3}\left(\mathrm{CH}_{2}\right)_{3} \mathrm{NH}_{3} \mathrm{CuCl}_{4}$ crystal for the heating run.

As seen in Fig. 2, the measured DTA dependence shows a clear anomaly at about $436 \mathrm{~K}$ connected with the first order phase transition. There is no anomaly at about $334 \mathrm{~K}$. This is in agreement with DSC studies. Thermal stability is seen well up to about $480 \mathrm{~K}$. The loss of mass observed at $480 \mathrm{~K}$ is connected with decomposition of the samples. Thus, the anomaly at $436 \mathrm{~K}$ observed in DSC and DTA shows evidence for the structural phase transition appearance in this crystal and it means that the phase transition observed in DSC is reversible.

The chemical analysis showed the following mass percentages of: (a) fresh sample: $\mathrm{C}-12.9 \pm 0.1, \mathrm{~N}-$ $10.05 \pm 0.1$, and $\mathrm{H}-4.3 \pm 0.1$, (b) heated sample: $\mathrm{C}-$ $12.9 \pm 0.1, \mathrm{~N}-10.02 \pm 0.1, \mathrm{H}-4.3 \pm 0.1$. Calculated percentages were: $\mathrm{C}-12.79, \mathrm{~N}-9.95, \mathrm{H}-4.26$. A chemical analysis did not show any noticeable changes of the composition after heating the crystal up to $450 \mathrm{~K}$ in comparison to the as-grown sample. This is in agreement with the TGA experiment where the loss of mass was not observed. Thus, the chemical analysis confirmed the thermal stability of the substance up to at least $450 \mathrm{~K}$. To get additional information about the reversibility of the phase transition, microscopic observations of the sample were done. Results of optical observations of as-grown fresh samples along the $b$-axis are presented in Fig. 3 . 

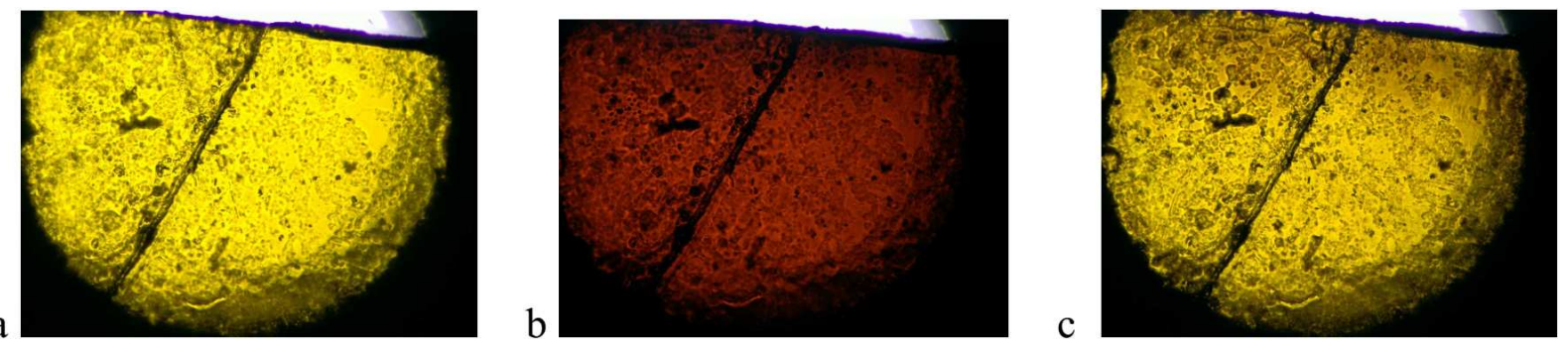

Fig. 3. Optical behaviour of the crystal observed by means of polarized microscopy.

On heating, the sample changes colour from yellow (Fig. 3a, $T=293 \mathrm{~K}$ ) to brown (Fig. 3b, $T=434 \mathrm{~K}$ ) in a continuous way. The phase transition does not cause any sharp change in the colour of the sample. After cooling down to room temperature the sample becomes yellow again, however a little darker (Fig. 3c, $T=300 \mathrm{~K}$ ). The sample that was covered with silicone oil exhibited an unchanged yellow colour, therefore the gradual darkening of the sample is caused mainly on the surface [10].

\subsection{Dielectric studies}

Dielectric properties of the $\mathrm{NH}_{3}\left(\mathrm{CH}_{2}\right)_{3} \mathrm{NH}_{3} \mathrm{CuCl}_{4}$ (DAPCCl) crystal obtained for chosen frequencies on heating/cooling runs are presented in Figs. 4 and 5.

Details of the dielectric response for the frequency $1 \mathrm{kHz}$ are presented in Fig. 5 .

As presented in Fig. $5 \mathrm{a}$ at the frequency $1 \mathrm{kHz}$ on heating the relative permittivity $\varepsilon^{\prime}$ is nearly constant up to about the phase transition temperature (433 K). At the phase transition range and above, the permittivity increases rapidly. Similar behaviour is observed in the case of losses $\varepsilon^{\prime \prime}$. Losses increase up to the phase transition temperature and at the phase transition region, losses diminish in a range of about $2 \mathrm{~K}$ and then increase rapidly above $435 \mathrm{~K}$. Thus, the detailed dependence of permittivity and losses observed in the range of the phase transition presented in Fig. 5 show a rather complex behavior.

Such a significant increase of permittivity and losses cannot be regarded as a ferroelectric phase transition as proposed in [8]. The crystal is centrosymmetric in both phases [10] thus it excludes ferroelectricity. The room temperature space group is orthorhombic Pnma but the symmetry of the phase above $433 \mathrm{~K}$ is monoclinic $B 2 / m$ [10]. The high value of the permittivity and losses result from the huge increase of conductivity. It is necessary to mention that there is no anomaly of the permittivity and losses on heating at about $334 \mathrm{~K}$. One unexpected observation is the remarkable difference of the temperature dependence of the permittivity and losses on cooling in comparison to the heating run. At lower frequencies, the permittivity increases further at the beginning of the cooling run and then diminishes. The losses reach huge values at lower frequencies. The phase transition is seen clearly on cooling both in permittivity

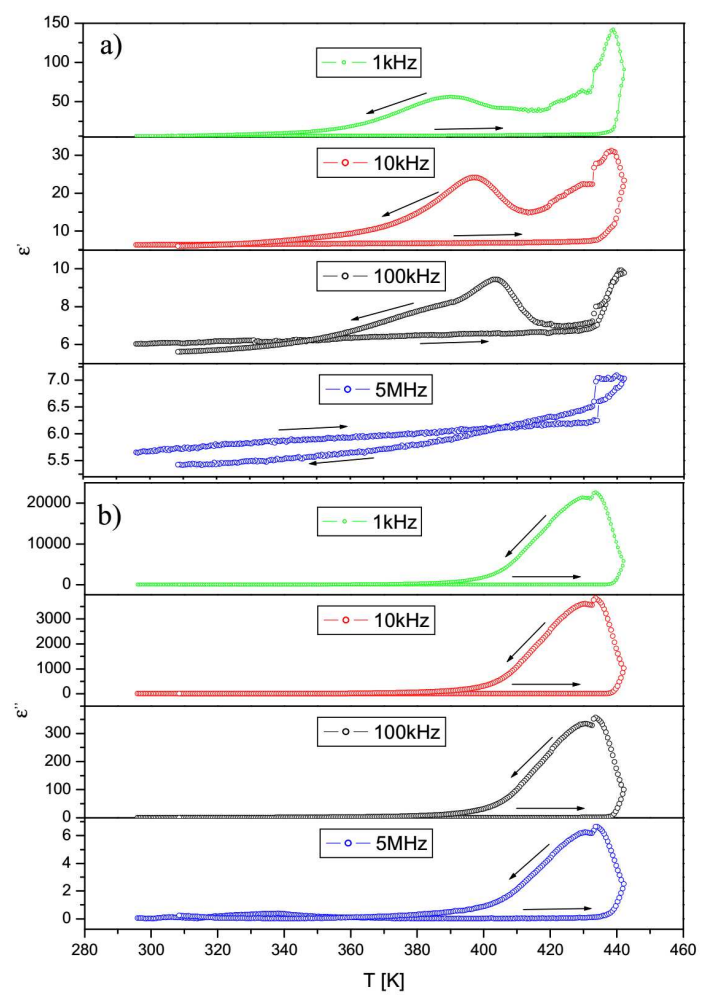

Fig. 4. Temperature dependence of the permittivity (a) and losses (b) for the $\mathrm{NH}_{3}\left(\mathrm{CH}_{2}\right)_{3} \mathrm{NH}_{3} \mathrm{CuCl}_{4}$ crystal for the heating/cooling runs for chosen frequencies.

and losses dependence. Additionally, on cooling, broad humps are seen in the range $420-340 \mathrm{~K}$. At other frequencies the dielectric behaviour is similar, but the values of permittivity and losses diminish on cooling with an increase of frequency (Fig. 4).

On cooling, the maxima of the humps shift to higher temperatures with increasing frequencies and disappear at $5 \mathrm{MHz}$. It is difficult to explain such dielectric behaviour and it is necessary to discuss the problem of the reversibility of the observed phase transition again. DSC measurements did not display any remarkable changes of thermal properties and proved the reversible phase transition. Chemical analysis did not show any change of the chemical composition of the compound. In addition, the TGA measurements did not exhibit any losses of mass on 

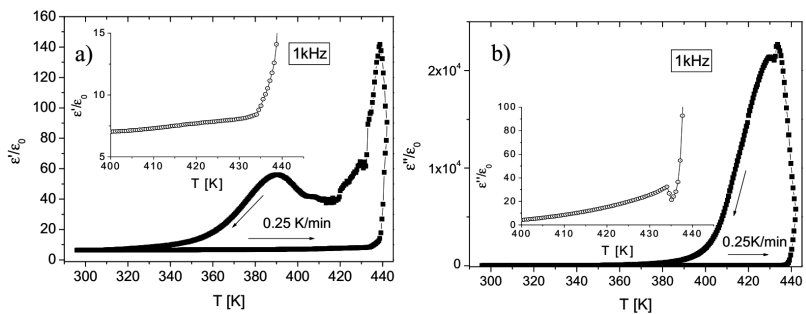

Fig. 5. Temperature dependence of the permittivity (a) and losses (b) for the crystal $\mathrm{NH}_{3}\left(\mathrm{CH}_{2}\right)_{3} \mathrm{NH}_{3} \mathrm{CuCl}_{4}$ for the heating/cooling runs for the frequency $1 \mathrm{kHz}$.

heating up to $480 \mathrm{~K}$. No significant changes of the sample after heating/cooling cycles were observed by polarized microscopy. X-ray studies showed a reversible change of symmetry observed upon heating and cooling, although with a decrease of line intensity on cooling run [10]. Thus, the observed dielectric response is probably connected with the creation and rearrangement of defects above the phase transition temperature that influence electric properties (space charge) of the material above the phase transition.

In spite of the chemical stability of the crystal (480 K) the temperature of $436 \mathrm{~K}$ is high enough to induce some destruction of the crystal lattice. These changes are not completely removed on cooling. Most probably, after a phase transition on heating, the long-time relaxation processes are present and they cause the strange dielectric behaviour. The phase transition is clearly seen at a frequency of $5 \mathrm{MHz}$ (Fig. 4a) due to the fact that at higher frequencies the contribution of the defected structure is much smaller than those at lower frequencies. The value of the permittivity at room temperature (after cooling) is quite similar to the initial value (before heating).

Similar dielectric behaviour was observed in the case of other hybrid crystals i.e. in pyrazine fluoroborate [11] and pyrazine perchlorate [12].

In our description of the dielectric response of the crystal we analyzed the results of the dielectric measurements obtained during heating. Taking into account the large changes of the dielectric properties connected with an increase of the conductivity we analyzed the results in terms of a complex impedance

$$
Z^{*}=Z^{\prime}-\mathrm{i} Z^{\prime \prime}
$$

and its frequency dependence

$$
Z^{*}(\omega)=\frac{Z_{s}}{1+\left(\mathrm{i} \omega \tau_{c}\right)^{\alpha}},
$$

where $Z_{s}$ is the bulk resistance, $\omega=2 \pi \nu$ is the angular frequency of the measuring field, $\tau_{c}=Z_{s} C_{s}$ is a time constant, and $C_{s}$ is the geometric capacity.

The frequency dependence of $Z^{\prime}$ and $Z^{\prime \prime}$ for various temperatures are presented in Fig. 6 .

As presented in Fig. 6, $Z^{\prime}$ decreases with increasing frequency and temperature in the range of 421-433 K (in phase I). A significant growth of $Z^{\prime}$ at a temperature $433.6 \mathrm{~K}$ can be regarded as the starting point of the phase transition.
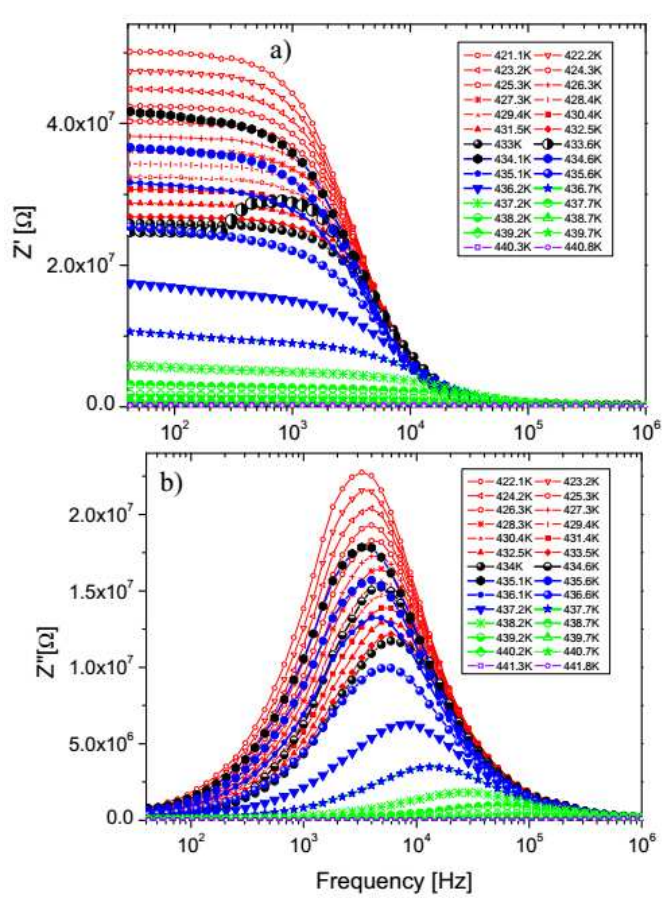

Fig. 6. Frequency dependence of $Z^{*}$ at various temperatures: (a) $Z^{\prime}$, (b) $Z^{\prime \prime}$ along the $b$-axis for the $\mathrm{NH}_{3}\left(\mathrm{CH}_{2}\right)_{3} \mathrm{NH}_{3} \mathrm{CuCl}_{4}$ crystal.

$Z^{\prime \prime}$ shows symmetrical maxima that shift to higher frequency with an increase of temperature. The maxima diminish with increasing temperature in phase I on approaching the phase transition temperature at $433 \mathrm{~K}$. It is interesting to note that the increase of the impedance $Z^{*}$ is observed just after the phase transition temperature in a narrow range of about $2 \mathrm{~K}$. Above $435.5 \mathrm{~K}$, $Z^{*}(T)$ exhibits behaviour similar to the dependence in the range $421-433 \mathrm{~K}$.

The plots of $Z^{\prime}$ versus $Z^{\prime \prime}$ for chosen temperatures around the phase transition temperature obtained from experimental results are presented in Fig. 7.

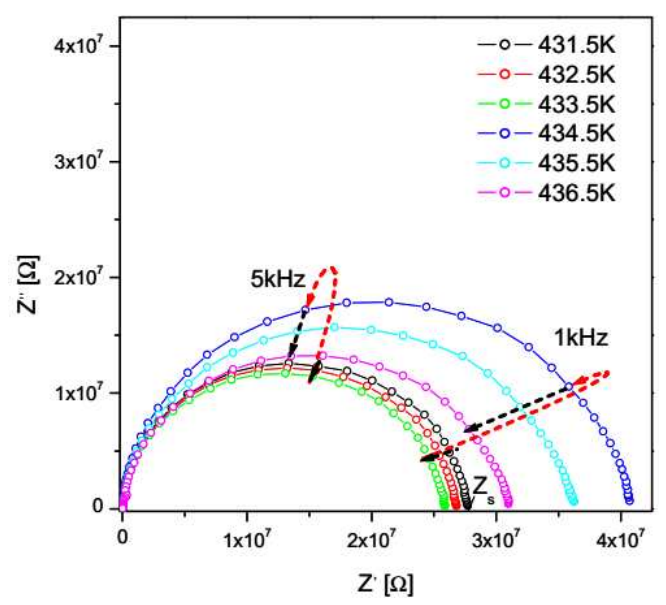

Fig. 7. The plots of $Z^{\prime \prime}$ versus $Z^{\prime}$ for the $\mathrm{NH}_{3}\left(\mathrm{CH}_{2}\right)_{3} \mathrm{NH}_{3} \mathrm{CuCl}_{4}$ crystal. 
At a temperature of $434.5 \mathrm{~K}$ a rapid growth of $Z_{s}$ was recorded which then diminished until $436.5 \mathrm{~K}$.

As shown in Fig. 7, these relations are presented as semicircles with approximately one relaxation time. The $\alpha$ coefficient is approximately one in the measured temperature range on both sides of the phase transition temperature. In the temperature range of 431-433 K the $\alpha$ coefficient value is 0.95 and is slightly lower $(0.92)$ in the range of $433-436 \mathrm{~K}$.

The $\sigma_{d c}$ conductivity is calculated from the bulk resistance $Z_{s}$, which itself is obtained from an intersection of semicircles with the $Z^{\prime}$ coordinates at the low frequency side. The temperature dependence of the $\sigma_{d c}$ conductivity is described using the Arrhenius equation

$$
\sigma_{d c}=\sigma_{0} \exp \left(-E_{a} / k_{\mathrm{B}} T\right)
$$

where $\sigma_{0}$ is the pre-exponential factor, $E_{a}$ is the activation energy, $k_{\mathrm{B}}$ is the Boltzmann constant, and $T$ is temperature.

On the other hand, from the maxima of semicircles presented in Fig. 7, one can find the conductivity relaxation time $\tau$ for different temperature ranges that are characteristic for the thermally activated hopping process [13], and can be described by the Arrhenius relation

$$
\tau=\tau_{0} \exp \left(-E_{a} / k_{\mathrm{B}} T\right),
$$

where $\tau_{0}$ is the pre-exponential factor.

The temperature dependence of $\ln \sigma_{d c}$ and $\ln \tau$ versus 1000/T are presented in Fig. 8.
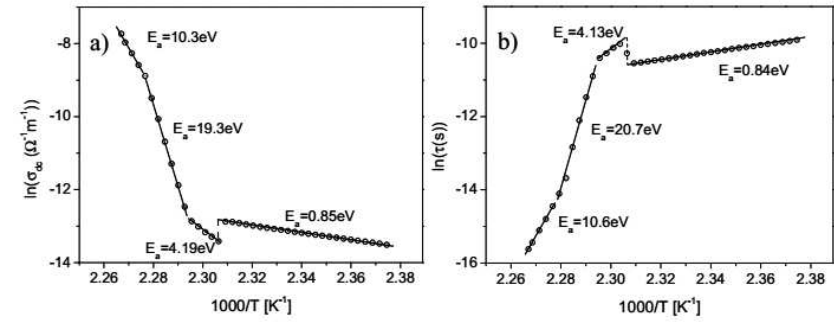

Fig. 8. Temperature dependence of $\ln \sigma_{d c}$ (a) and $\ln \tau$ (b) versus $1000 / T$ for the $\mathrm{NH}_{3}\left(\mathrm{CH}_{2}\right)_{3} \mathrm{NH}_{3} \mathrm{CuCl}_{4}$ crystal.

From this dependence, one can distinguish at least three temperature ranges with different activation energy. In the range of 421-433 $\mathrm{K}$ the activation energy is equal to $0.85 \mathrm{eV}$. One can see that at $433.5 \mathrm{~K}$ the $E_{a}$ increases and in the range of about $2 \mathrm{~K}$ the activation energy is equal to $4.19 \mathrm{eV}$. This $E_{a}$ value is much higher, at approximately five times the activation energy below the phase transition temperature. This narrow range of $2 \mathrm{~K}$ corresponds to that of the temperature where the observed domain structure exists as was seen by polarized microscopy. In the case of natural twinned samples domains do not vanish during the first order transition after the phase front passing through the crystal on heating. However, new domains perpendicular to existing domains appear at the transition region, and they exist in the range $2-3 \mathrm{~K}$ above the phase transition temperature and then they disappear. This is similar to domain appearance in monodomain samples existing in the temperature range $2-3 \mathrm{~K}$ above the phase transition temperature [10].

The unusual growth of $Z_{s}$ and then diminishing was observed at the same narrow temperature range of $2 \mathrm{~K}$ above the phase transition temperature (Fig. 7).

In the range of 436-440 $\mathrm{K}$ the activation energy increases again to $19.3 \mathrm{eV}$ and then diminishes to the value of $10.3 \mathrm{eV}$.

Similar changes of the activation energy (increase of $E_{a}$ with increase of temperature) were observed in the case of powdered sample [8].

An increment of the activation energy in the range of 2-3 K above the transition temperature can be connected with the coexistence and competition between two domain structures of mutually perpendicular domain walls. A further increase of the activation energy can be influenced by the layered domain structure that appears above the phase transition temperature, with domain walls perpendicular to the measuring field, and also by the presence of defects.

\subsection{PAL studies}

In order to better clarify this strange phase transition, we performed an additional experiment (PAL). We studied the fresh-grown crystal and the crystal heated up to $443 \mathrm{~K}$ and cooled to room temperature to see if the heating process causes changes in the defect structure. This method is a sensitive tool for the investigation of defects in solids. The trapping of the positron in defects is based on the formation of an attractive potential at open-volume defects, such as vacancies, vacancy agglomerates, and dislocations. When the positron is trapped in an open-volume defect, the annihilation parameters are changed in a characteristic way. The positron lifetime increases in an open-volume defect due to the lower electron density. The analysis of the annihilation radiation thus gives the possibility of defect detection. A clustering of defects may be observed as the increase in the defect-related lifetime due to the further decrease in the electron density.

The applied deconvolution procedure for PAL spectra is assumed to cover independent channels of annihilation in defect-free bulk and trapping in one type of spatially extended free-volume defects. Numerical characteristics of positron trapping such as mean $\tau_{a v}$, defect-related $\tau_{2}$, defect-free bulk $\tau_{b}$ positron lifetimes and positron trapping rate in defect $\kappa_{d}$ were parameterized in respect of formalism of the known two-state positron trapping model [14]:

$$
\begin{aligned}
\tau_{a v} & =\tau_{1} I_{1}+\tau_{2} I_{2}, \\
\tau_{b} & =\frac{\tau_{1} \tau_{2}}{\tau_{2} I_{1}+\tau_{1} I_{2}} \\
\tau_{d} & =\tau_{2}, \\
\kappa_{d} & =\frac{I_{2}}{I_{1}}\left(\frac{1}{\tau_{b}}-\frac{1}{\tau_{2}}\right) .
\end{aligned}
$$


TABLE I

PAL parameters for the $\mathrm{NH}_{3}\left(\mathrm{CH}_{2}\right)_{3} \mathrm{NH}_{3} \mathrm{CuCl}_{4}$ crystal at the room temperature. Standard deviations are given in round brackets.

\begin{tabular}{c|c|c|c|c|c|c|c}
\hline \hline & $\tau_{1}[\mathrm{ps}]$ & $\mathrm{I}_{1}[\%]$ & $\tau_{2}[\mathrm{ps}]$ & $\mathrm{I}_{2}[\%]$ & $\tau_{a v}[\mathrm{ps}]$ & $\tau_{b}[\mathrm{ps}]$ & $\kappa_{d}\left[\frac{1}{\mathrm{~ns}}\right]$ \\
\hline before & 207.7 & 80.5 & 358.7 & 19.5 & 237.0 & 226 & 1.759 \\
heating & $(2.2)$ & $(1.6)$ & $(7.8)$ & $(1.6)$ & $(6.9)$ & $(16)$ & $(0.085)$ \\
\hline after & 209.3 & 87.66 & 422.0 & 12.34 & 235.3 & 223.1 & 0.970 \\
cooling & $(1.1)$ & $(0.66)$ & $(7.0)$ & $(0.66)$ & $(3.3)$ & $(8.5)$ & $(0.029)$
\end{tabular}

The results obtained before heating and after cooling, for measurements at room temperature, are presented in Table I.

Analysis of the measurement data gave the best results for the decomposition of spectra into two components of mean lifetimes $\tau_{1}$ and $\tau_{2}$ and intensities $I_{1}$ and $I_{2}$, respectively. The fit parameter $\left(\chi^{2}\right)$ was less than 0.912 for each of the analyzed spectra. According to the trapping model that was used, annihilations occur at defects or free volume of one type $\left(\tau_{2}\right)$ and in the bulk $\left(\tau_{b}\right)$. The increase of the lifetime of the positrons annihilating within defects $\left(\tau_{2}\right)$ from 358.7 ps to 422.0 ps makes evident the increase of the defect size. The decrease of the intensity of the defect component $I_{2}$ from $19.5 \%$ to $12.34 \%$ may be associated with the defect conglomeration during heating. After cooling, the residues of these conglomerations can still exist in the sample. The changes of the defect state before and after heating, observed at the room temperature by PAL method, can also be clearly seen by optical studies where a reduction of sample transparency has taken place (see Fig. 3)

The reduction of $\kappa_{d}$ from $1.759 \mathrm{~ns}^{-1}$ to $0.970 \mathrm{~ns}^{-1}$ for the samples before heating and after cooling could be due to an increase of the dimensions of defects signaled by the increase of $\tau_{2}$. It may also be due to an increase of the electrical potential in the defect areas, which affect the positron trapping rate $\left(\kappa_{d}\right)$. The changes of the annihilation parameters, such as $\tau_{2}, \mathrm{I}_{2}, \kappa_{d}$ indicate the possibility of influencing the sample properties by applying a thermal stimulation. However, heating and cooling of the samples did not significantly influence the values of $\tau_{a v}$ and $\tau_{b}$ therefore confirming the absence of changes in the crystal structure. The similarity of the average lifetime for the samples before and after heating does not exclude the possibility of changes in the value of $\tau_{2}$. In Fig. 9 the slight differences in the defect component are visible.

The spectra presented in Fig. 9 are normalized to equal number of counts in the range of time from $-1.041 \mathrm{~ns}$ (start channel) to $5.259 \mathrm{~ns}$. As can be seen in the picture, particularly from $1.5 \mathrm{~ns}$ to $3 \mathrm{~ns}$, the red circles that are arranged along the line are more gently sloped in comparison with the blue line. The slope of the line determines the value of $\tau_{2}$, thereby clearly showing the different values of $\tau_{2}$ before heating $\left(\tau_{2}=358.7 \mathrm{~ns}\right)$ and after heating and then cooling $\left(\tau_{2}=422.0 \mathrm{~ns}\right)$.

Possible changes can be related to clustering of defects. Changes in the defected structure at room temperature after cooling from high-temperature phase lead

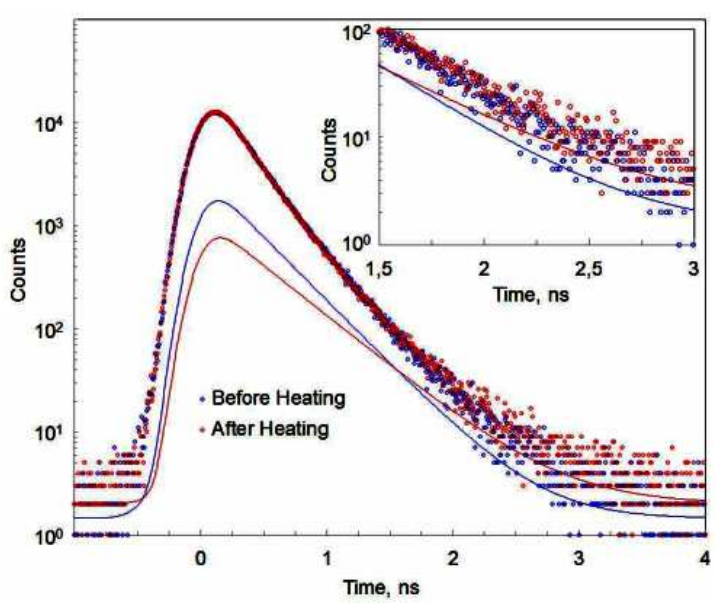

Fig. 9. The positron annihilation lifetime spectra for the $\mathrm{NH}_{3}\left(\mathrm{CH}_{2}\right)_{3} \mathrm{NH}_{3} \mathrm{CuCl}_{4}$ crystal before (blue circles) and after the heat treatment (red circles). The theoretical data of the second component, presented on the picture as lines, were calculated on the basis of the trapping model by the LT9 program. The blue line refers to the measurement prior to heating, the red line - after heating.

to the conclusion that these changes must be significant at higher temperatures. Unfortunately, the PAL spectra could be obtained only at the room temperature. In spite of the fact that the crystal is now at room temperature again, thermal equilibrium is seemingly not achieved, a consequence of changes induced at higher temperatures (mainly above the phase transition temperatures). One should also remember that the electric permittivity returns to the initial value at room temperature.

\section{Summary}

1. The crystal of $\mathrm{NH}_{3}\left(\mathrm{CH}_{2}\right)_{3} \mathrm{NH}_{3} \mathrm{CuCl}_{4}$ undergoes the first order-phase transition at $436 / 434 \mathrm{~K}$ on heating/cooling runs observed by DSC method; the change of entropy at the transition indicates the displacive type of the phase transition;

2. TGA and DTA studies show thermal stability of the substance up to $480 \mathrm{~K}$ and confirm the structural phase transition at $435 \mathrm{~K}$ on heating run;

3. Dielectric studies confirm the phase transition at $434 \mathrm{~K}$ and showed a strongly hysteretic behaviour probably connected with the appearance of the space charge and the rearrangement of defects induced during heating above the phase transition;

4. Some changes in the "defected structure" after heating were observed in PAL studies;

5. The activation energy depends strongly on the temperature and is much higher in those ranges that are above the phase transition temperature. 


\section{References}

[1] L.P. Battaglia, A. Bonamartini Corradi, G. Pelosi, M.R. Cramarossa, T. Manfredini, G.C. Pellacani, A. Motori, A. Saccani, F. Sandrolini, M.F. Brigatti, Chem. Matter 4, 813 (1992).

[2] R. Blinc, M. Burgar, B. Lozar, J. Seliger, J. Slak, V. Rutar, H. Arend, R. Kind, J. Chem. Phys. 66, 278 (1977).

[3] B. Staśkiewicz, I. Turowska-Tyrk, J. Baran, Cz. Górecki, Z. Czapla, J. Phys. Chem. Solids 75, 1305 (2014).

[4] H. Ishihara, Shi-qi Dou, K. Horiuchi, V.G. Krishnan, H. Paulus, H. Fuess, A. Weiss, Z. Naturforsch. a 51, 1216 (1996).

[5] B. Staśkiewicz, O. Czupiński, Z. Czapla, J. Mol. Struct. 1074, 723 (2014).

[6] R. Kind, S. Plesko, J. Ross, Phys. Status Solidi A 47, 233 (1978).
[7] D. Phelps, D. Losee, W. Hatfield, D. Hodgson, Inorg. Chem. 15, 3147 (1976).

[8] M.F. Mostafa, A.A. Youssef, S.S. Montasser, S.S. Khyami, Z. Naturforsch. a 60, 837 (2005).

[9] J. Kansy, Nucl. Instrum. Methods Phys. Res. A 374, 235 (1996).

[10] Z. Czapla, J. Przesławski, M. Crofton, J. Janczak, O. Czupiński, A. Ingram, M. Kostrzewa, Phase Transitions, 1252983 (2016).

[11] A. Katrusiak, M. Szafrański, J. Am. Chem. Soc. 128, 15775 (2006).

[12] M. Szafrański, M. Jarek, Cryst. Eng. Commun. 15, 4617 (2013).

[13] A.K. Jonscher, Dielectric Relaxation in Solids, Chelsea Dielectric Press, London 1983, p. 340.

[14] R. Krause-Rehberg, H.S. Leipner, Positron Annihilation in Semiconductors. Defect Studies, SpringerVerlag, Berlin 1999 